\title{
Real sets
}

\author{
George Janelidze* \\ Dept Math. \& Appl. Math., University of Cape Town, Rondebosch 7701, South Africa \\ $<$ george.janelidze@uct.ac.za> \\ Ross Street $^{\dagger}$ \\ Centre of Australian Category Theory, Macquarie University, NSW 2109, Australia \\ $<$ ross.street@mq.edu.au $>$ \\ January 17, 2018
}

Dedicated to Peter Freyd and Bill Lawvere.

2010 Mathematics Subject Classification: 18D10; 18D20; 20M14; 28A20

Key words and phrases: commutative monoid; biproduct; direct sum; abstract addition; magnitude module; series monoidal category.

\begin{abstract}
After reviewing a universal characterization of the extended positive real numbers published by Denis Higgs in 1978, we define a category which provides an answer to the questions:

- what is a set with half an element?

- what is a set with $\pi$ elements?

The category of these extended positive real sets is equipped with a countable tensor product. We develop somewhat the theory of categories with countable tensors; we call the commutative such categories series monoidal and conclude by only briefly mentioning the non-commutative possibility called $\omega$-monoidal. We include some remarks on sets having cardinalities in $[-\infty, \infty]$.
\end{abstract}

*This author gratefully acknowledges the support of the South African National Research Foundation.

†This author gratefully acknowledges the support of Australian Research Council Discovery Grants DP1094883, DP130101969 and DP160101519. 


\section{Contents}

1 Introduction 2

2 Series magmas and series monoids 3

3 The symmetric closed structure $\quad 8$

4 Zeno morphisms and magnitude modules 11

5 Series monoidal categories 13

6 Zeno functors and magnitude categories 21

$\begin{array}{llr}7 & \text { Remarks on integer sets } & 26\end{array}$

8 Categories enriched in a series $\begin{array}{ll}\text { monoidal category } & 27\end{array}$

9 w-Magmas and $\omega$-monoids $\quad 28$

$10 \omega$-Monoidal categories $\quad 29$

That which is in locomotion must arrive at the half-way stage before it arrives at the goal. Zeno [1]

\section{Introduction}

For many years the authors drafted joint notes on a general project dedicated to developing the theory of categories with tensor products of infinitely many objects. As part of that, we were interested in sets with infinite operations. There is already some literature in this direction: for example, Tarski's book [28], and the work starting with Linton and Semadeni [23] and leading to a series of papers including Fillmore-Pumplün-Röhrl [7].

Serendipity led us recently to Higgs' paper [9] which provides a universal property for the set $[0, \infty]$ of extended positive real numbers with structure involving infinite summation. The paper acknowledges ideas of Huntingdon [11] and Tarski [28]. More importantly for the current Special Volume is Higgs' interesting paragraph which begins with the sentence:

In conclusion, I would like to say that the stimulus for the introduction of magnitude modules was a question of Lawvere as to whether a direct definition 
of the continuum, appropriate for use in a topos, could be given.

Also, of course, Bill Lawvere [19] used $[0, \infty]$ as a base for recognizing metric spaces as a fertile part of category theory.

Moreover, as the unary operation of halving is used by Higgs to pin down $[0, \infty]$, surely there are connections with the work of Peter Freyd [8] which involves the mid-point operation. Such relationships, apart from the fact that real intervals are involved, are not yet apparent to the authors.

Consequently, [9] was the trigger for us to focus our infinite tensor work on deciding what might be a set with a real cardinality. The first four sections of the present paper are essentially a reorganization of Higgs' paper, emphasising the structures we later use to provide our categorical version.

In Section 5, we define series monoidal categories as categories equipped with a countable summation operation appropriately axiomatized. Many examples are explained. What we call Zeno functors in Section 6 allow us to halve objects; these endofunctors universally lead to our category of extended positive real sets.

The logarithm of a positive real may be negative. Section 7 mentions that and other ideas about capturing all real numbers and sets.

One of the purposes of symmetric monoidal categories is to serve as bases for enriched categories. In Section 8, we look at categories enriched in a series monoidal category and show that they form a series monoidal 2-category. On the excuse that one of our constructions could lead us to a non-symmetric example, we briefly look in the last Sections 9 and 10 at non-symmetric infinitary operations.

We suspect the reason no one has suggested our construction of the category of positive real sets is that the Higgs paper was looked at more for its contribution to measure theory [10] and that categories with infinite tensor products have not had much attention.

Note: In Section 5 we explain that Examples 5.5, 5.6, 5.12, and 5.13 in the published version [Real sets, Tbilisi Math. J. 10(3) (2017) 23-49] were incorrect.

\section{Series magmas and series monoids}

Let $\mathbb{N}$ denote the natural numbers which include 0 . For sets $X$ and $A$, we write $A^{X}$ for the set of functions $a: X \rightarrow A$ and we often put $a_{x}:=a(x)$ and $\left(a_{x}\right)_{x \in X}:=a$. Given $0 \in A$, define

$$
\delta: A \longrightarrow A^{\mathbb{N} \times \mathbb{N}}
$$


by

$$
\delta(a)_{m, n}= \begin{cases}a & \text { if } m=n \\ 0 & \text { if } m \neq n\end{cases}
$$

We identify $\delta$ with its composite with either of the canonical isomorphisms $\sigma_{1}, \sigma_{2}: A^{\mathbb{N} \times \mathbb{N}} \cong\left(A^{\mathbb{N}}\right)^{\mathbb{N}}$, where

$$
\sigma_{1}(a)(m)(n)=a_{m, n}=\sigma_{2}(a)(n)(m),
$$

since $\sigma_{1} \circ \delta=\sigma_{2} \circ \delta$. We also write $\delta_{n}: A \rightarrow A^{\mathbb{N}}$ for $a \mapsto\left(\delta(a)_{m, n}\right)_{m \in \mathbb{N}}$.

Definition 2.1. A series magma is a set $A$ equipped with an element $0 \in A$ and a function

$$
\Sigma: A^{\mathbb{N}} \longrightarrow A,\left(a_{i}\right)_{i \in \mathbb{N}} \longmapsto \Sigma_{i \in \mathbb{N}} a_{i}
$$

such that the following diagram commutes for all $n \in \mathbb{N}$.

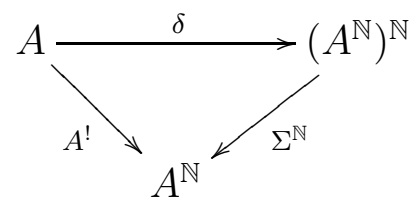

For any series magma $A$ and subset $S \subseteq \mathbb{N}$, we can define an operation $\Sigma_{S}: A^{S} \rightarrow A$ whose value at $a \in A^{S}$ is

$$
\Sigma_{n \in S} a_{n}=\Sigma_{n \in \mathbb{N}} c_{n}
$$

where

$$
c_{n}= \begin{cases}a_{n} & \text { if } n \in S \\ 0 & \text { otherwise } .\end{cases}
$$

Since series magmas are models of an algebraic theory, there is a corresponding notion of morphism, that is, a function $f: A \rightarrow B$ such that $f(0)=0$ and the following square commutes.

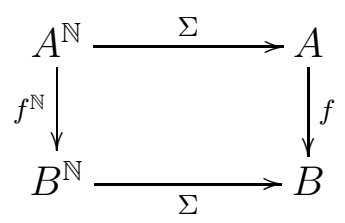

Also, the resultant category SerMg of series magmas is both complete and cocomplete, and is Barr-Tierney exact. The forgetful functor U: SerMg $\rightarrow$ 
Set is monadic. The monad generated by $U$ and its left adjoint preserves $\aleph_{1}$-filtered colimits.

An aspect of all this is that $A^{\mathbb{N}}$ is the underlying set for the cotensor of the set $\mathbb{N}$ with the series magma $A$; the series magma structure consists of the constant sequence $0=\delta(0)_{0}$ and $\Sigma^{\mathbb{N}}:\left(A^{\mathbb{N}}\right)^{\mathbb{N}} \rightarrow A^{\mathbb{N}}$.

Here is an easy Eckmann-Hilton-type result.

Proposition 2.2. Suppose a set $A$ has two series magma structures $\Sigma$ and $\Sigma^{\prime}$ with the same 0 . If $\Sigma^{\prime}: A^{\mathbb{N}} \rightarrow A$ is a morphism for the $\Sigma$ structure on $A$ then $\Sigma^{\prime}=\Sigma$ and, for all $a \in A^{\mathbb{N} \times \mathbb{N}}$,

$$
\Sigma_{m \in \mathbb{N}} \Sigma_{n \in \mathbb{N}} a_{m, n}=\Sigma_{n \in \mathbb{N}} \Sigma_{m \in \mathbb{N}} a_{m, n} .
$$

Proof. The morphism condition (2.3) for $\Sigma^{\prime}$ is

$$
\Sigma_{m \in \mathbb{N}}^{\prime} \Sigma_{n \in \mathbb{N}} a_{m, n}=\Sigma_{n \in \mathbb{N}} \Sigma_{m \in \mathbb{N}}^{\prime} a_{m, n} .
$$

In this, for any $b \in A^{\mathbb{N}}$, take the diagonal matrix $a_{m, n}=\delta\left(b_{m}\right)_{m, n}$. Using (2.1) for both sums, we obtain $\Sigma_{m \in \mathbb{N}}^{\prime} b_{m}=\Sigma_{n \in \mathbb{N}} b_{n}$; that is, $\Sigma^{\prime}(b)=\Sigma(b)$.

Definition 2.3. A series monoid is a series magma satisfying (2.4). Write SerMn for the full subcategory of SerMg consisting of the series monoids.

Example 2.4. The natural numbers $\mathbb{N} \cup\{\infty\}$, extended to include $\infty$, is a series monoid with 0 the natural number 0 and

$$
\Sigma_{n \in \mathbb{N}} a_{n}= \begin{cases}\sum_{n=0}^{\infty} a_{n} & \text { if } a \text { has finite support } \\ \infty & \text { otherwise } .\end{cases}
$$

Example 2.5. Similarly, the non-negative real numbers $[0, \infty]$, extended to include $\infty$, is a series monoid with 0 the real number 0 and

$$
\Sigma_{n \in \mathbb{N}} a_{n}= \begin{cases}\sum_{n=0}^{\infty} a_{n} & \text { if the series converges } \\ \infty & \text { otherwise }\end{cases}
$$

Example 2.6. For any series monoid $A$ and any set $X$, there is the pointwise series monoid structure on $A^{X}$. For various choices of $X$ and $A$, there can be interesting series submonoids of $A^{X}$. With $X$ a measurable space and $A=[0, \infty]$, the measurable functions $f: X \rightarrow[0, \infty]$ form a series submonoid of $[0, \infty]^{X}$. With $X=A=[0, \infty]$, the continuous non-decreasing functions form a series submonoid of $[0, \infty]^{[0, \infty]}$.

Example 2.7. Any partially ordered set $A$ admitting countable suprema is a series monoid with 0 the bottom element and $\Sigma$ equal to the countable supremum operation $\bigvee$. 
Proposition 2.8. Suppose $A$ is a series monoid and $\xi: \mathbb{N} \rightarrow \mathbb{N}$ is an injective function. If $a \in A^{\mathbb{N}}$ is such that $a_{n}=0$ for $n$ not in the image of $\xi$, then $\Sigma_{n} a_{\xi(n)}=\Sigma_{n} a_{n}$

Proof. We define $b_{m, n}$ to be $a_{\xi(m)}$ for $n=\xi(m)$ and to be 0 otherwise. Since $\xi$ is injective, each row and column of the matrix $b$ has at most one non-zero entry. Applying (2.4) to $b$ and using (2.1), we obtain the result.

Remark 2.9. Similarly, if $\xi: \mathbb{N} \rightarrow \mathbb{N} \times \mathbb{N}$ is an injective function and $a_{m, n}=0$ for $(m, n)$ not in the image of $\xi$, then $\Sigma_{n} a_{\xi(n)}=\Sigma_{(m, n)} a_{(m, n)}$ where, of course, the right-hand side is either side of (2.4). We leave this as an exercise.

As a particular case of (2.2), we can define a binary operation $a_{1}+a_{2}=$ $\Sigma_{n \in\{1,2\}} a_{n}$. This makes the series monoid $A$ into a commutative monoid with 0 as identity for + . Moreover, $\Sigma: A^{\mathbb{N}} \rightarrow A$ is a monoid morphism. The informal notation

$$
\Sigma_{n} a_{n}=a_{0}+a_{1}+a_{2}+\ldots
$$

can be suggestive.

We can also make $A$ into a pre-ordered set by defining $a \leq b$ when there exists $u$ with $a+u=b$. It is clearly reflexive, transitive, has 0 as least element, and is respected by $\Sigma$.

Definition 2.10. A series monoid is called idempotent when, for all $c \in A$ and $a \in A^{\mathbb{N}}$ such that $a_{n} \neq 0$ implies $a_{n}=c$, it follows that $\Sigma_{n} a_{n}=c$ holds.

Proposition 2.11. A series monoid arises from a partially ordered set as in Example 2.7 if and only if it is idempotent.

Proof. Suppose $A$ is an idempotent series monoid. We can prove that the order is antisymmetric. For, take $a \leq b$ and $b \leq a$; so we have $a+u=b$ and $b+v=a$. Then $a=a+u+v=a+(u+v)+(u+v)+(u+v)+\ldots$ by idempotence. So

$a=a+u+(v+u)+(v+u)+\cdots=a+(u+v)+(u+v)+\cdots+u=a+u=b$.

To see that $\Sigma_{n} a_{n}$ is the supremum of $\left\{a_{n}: n \in \mathbb{N}\right\}$, we have $a_{m}+\Sigma_{n} a_{n}=\Sigma_{n} a_{n}$ by commutativity and idempotency; so $a_{m} \leq \Sigma_{n} a_{n}$. Now suppose $a_{n} \leq c$ for all $n$. This means there exist $u_{n}$ with $a_{n}+u_{n}=c$ for all $n$. Since $\Sigma$ is a monoid morphism and because of idempotency, we have $\Sigma_{n} a_{n}+\Sigma_{n} u_{n}=c$. So $\Sigma_{n} a_{n} \leq c$.

The forgetful functor $\mathrm{U}:$ SerMn $\rightarrow$ Set has a left adjoint whose value at 1 can be made explicit. 
Proposition 2.12. The free series monoid on a single generating element is $\mathbb{N} \cup\{\infty\}$ as in Example 2.4. In other words, $\mathbb{N} \cup\{\infty\}$ is a representing object for the functor $\mathrm{U}$.

Proof. Given a series monoid $A$, we will show that

$$
\mathrm{ev}_{1}: \operatorname{SerMn}(\mathbb{N} \cup\{\infty\}, A) \rightarrow A
$$

taking $f$ to $f(1)$ is bijective. Take $a \in A$ and define $f_{a}: \mathbb{N} \cup\{\infty\} \rightarrow A$ by

$$
f_{a}(n)=n a=\overbrace{a+\cdots+a}^{n}
$$

and $f_{a}(\infty)=a+a+\ldots$ Then $f_{a}(1)=a$. Also, for $f_{a}: \mathbb{N} \cup\{\infty\} \rightarrow A$, we have $f_{f(1)}(n)=f(1)+\ldots f(1)=f(1+\cdots+1)=f(n)$ and $f_{f(1)}(\infty)=$ $f(1)+f(1) \cdots=f(1+1+\ldots)=f(\infty)$, so $f_{f(1)}=f$.

Countable products and sums (= coproducts) in SerMn are special: they coincide. We shall explain this although it is much like the case of finite direct products for commutative monoids.

Consider a sequence $\left(A_{k}\right)_{k \in \mathbb{N}}$ of series monoids. The cartesian product $\prod_{k \in \mathbb{N}} A_{k}$ becomes a series monoid by defining $\Sigma$ to be the composite

$$
\left(\prod_{k \in \mathbb{N}} A_{k}\right)^{\mathbb{N}} \cong \prod_{k \in \mathbb{N}}\left(A_{k}\right)^{\mathbb{N}} \stackrel{\prod_{k \in \mathbb{N}} \Sigma}{\longrightarrow} \prod_{k \in \mathbb{N}} A_{k} .
$$

The projections $\mathrm{pr}_{k}: \prod_{k \in \mathbb{N}} A_{k} \longrightarrow A_{k}$ are all morphisms of series monoids. This gives the product in the category SerMn.

Now, we can define morphisms $\operatorname{in}_{k}: A_{k} \longrightarrow \prod_{h \in \mathbb{N}} A_{h}$ by

$$
\operatorname{in}_{k}(a)_{h}= \begin{cases}a & \text { for } h=k, \\ 0 & \text { for } h \neq k\end{cases}
$$

Proposition 2.13. The family of morphisms $\operatorname{in}_{k}: A_{k} \longrightarrow \prod_{h \in \mathbb{N}} A_{h}$, for $k \in \mathbb{N}$, is a coproduct in the category SerMn. The following formulas hold:

$$
\begin{gathered}
\Sigma_{k \in \mathbb{N}} \operatorname{in}_{k} \circ \operatorname{pr}_{k}=1_{\prod_{h \in \mathbb{N}} A_{h}}, \\
\operatorname{pr}_{k} \circ \operatorname{in}_{m}= \begin{cases}1_{A_{k}} & \text { for } k=m, \\
0 & \text { for } k \neq m .\end{cases}
\end{gathered}
$$

Proof. The second sentence is an immediate consequence of the definitions. To prove we have a coproduct, take a family of morphisms $f_{k}: A_{k} \longrightarrow B$ into a series monoid $B$. Using the formulas of the second sentence, we deduce that the only morphism $f: \prod_{k \in \mathbb{N}} A_{k} \longrightarrow B$ with $f \circ \operatorname{in}_{k}=f_{k}$ for all $k \in \mathbb{N}$ is $f=\Sigma_{k \in \mathbb{N}} f_{k}$. 
For families $\left(A_{i}\right)_{i \in I}$ with $I$ not countable, the product is still the cartesian product with pointwise operations. The coproduct is the subobject consisting of the families of countable support. With this, it follows from Proposition 2.12 that we can describe all free series monoids (since free functors preserve coproducts and every set is a coproduct of one-element sets $1)$.

Proposition 2.14. The free series monoid on a set $X$ is the subobject of $(\mathbb{N} \cup\{\infty\})^{X}$ (as in Example 2.4) consisting of the functions of countable support.

\section{The symmetric closed structure}

For series monoids $A$ and $B$, we write $\operatorname{ser}(A, B)$ for the set $\operatorname{SerMn}(A, B)$ equipped with the pointwise series monoid structure. From Proposition 2.12, we have an isomorphism

$$
\mathrm{i}_{\operatorname{ser}(A, A)}:[\mathbb{N} \cup\{\infty\}, \operatorname{ser}(A, A)] \cong \operatorname{ser}(A, A),
$$

and so a morphism

$$
\mathrm{j}_{A}: \mathbb{N} \cup\{\infty\} \longrightarrow \operatorname{ser}(A, A)
$$

corresponding to the identity morphism $1_{A} \in \operatorname{ser}(A, A)$.

Since $\Sigma$ for each $\operatorname{ser}(C, D)$ is defined pointwise in $C$, we have a morphism

$$
\mathrm{L}^{A}: \operatorname{ser}(B, C) \longrightarrow \operatorname{ser}(\operatorname{ser}(A, B), \operatorname{ser}(A, C))
$$

defined by $\mathrm{L}^{A}(g)(f)=g \circ f$.

There is also an isomorphism

$$
\mathrm{s}_{A B C}: \operatorname{ser}(A, \operatorname{ser}(B, C)) \cong \operatorname{ser}(B, \operatorname{ser}(A, C))
$$

defined by noting that both sides are isomorphic to the pointwise series monoid of functions $f: A \times B \longrightarrow C$ for which all $f(a,-): B \longrightarrow C$ and $f(-, b): A \longrightarrow C$ are morphisms.

See [6] and [26] for the definition of closed category and the definition of category enriched in a closed category.

Proposition 3.1. A symmetric closed structure on the category SerMn is defined by $(\mathrm{i}, \mathrm{j}, \mathrm{L}, \mathrm{s})$. The obvious inclusions $1 \longrightarrow \mathbb{N} \cup\{\infty\}$ and $\mathrm{U}[A, B] \longrightarrow$ $(\mathrm{U} B)^{\mathrm{U} A}$ provide the forgetful functor $\mathrm{U}: \mathrm{SerMn} \longrightarrow$ Set with a closed structure. 
Proof. To check that the axioms pass from those axioms for the cartesian closed structure on Set we use the facts that each $\mathrm{U}[A, B] \longrightarrow(\mathrm{U} B)^{\mathrm{U} A}$ is a monomorphism, and that $\mathbb{N} \cup\{\infty\}$ is free on 1 (Proposition 2.12).

Proposition 3.2. The forgetful functor $\mathrm{U}$ : SerMn $\longrightarrow$ Set is monadic of rank $\aleph_{1}$. The left adjoint is defined on objects in Proposition 2.14. The monad on Set generated by the adjunction is closed (= monoidal).

Proof. The theory of series monoids is commutative.

By the general theory provided by Kock [18], the closed structure of Section 3 (see Proposition 3.1) is monoidal. We will write $A \otimes B$ for the tensor product of series monoids. We are interested in monoids for this tensor product; they might be called series rigs. (The term "rig" was used by Lawvere and Schanuel; the lack of an " $\mathrm{n}$ " in the word was to indicate the lack of negatives in the otherwise ring.)

Let $A$ be a commutative series rig; that is a commutative monoid in the symmetric monoidal category SerMn. We will write the operation of the monoid multiplicatively. This product distributes over $\Sigma$, and 0 acts as a zero. By associativity and commutativity, for each family $a=\left(a_{n}\right)_{n \in S}$ of elements of $A$ indexed by a finite set $S$, there is an element $\Pi_{m \in S} a_{m} \in A$.

Write $\left(\begin{array}{l}\mathbb{N} \\ n\end{array}\right)$ for the set of subsets of $\mathbb{N}$ of cardinality $n$.

Now for $a \in A^{\mathbb{N}}$, define

$$
\mathrm{P} a=\mathrm{P}_{r \in \mathbb{N}} a_{r}=\Sigma_{0<n \in \mathbb{N}} \Sigma_{S \in\left(\begin{array}{c}
\mathbb{N} \\
n
\end{array}\right)} \Pi_{m \in S} a_{m} .
$$

Less formally,

$$
\mathrm{P} a=\Sigma_{i} a_{i}+\Sigma_{i<j} a_{i} a_{j}+\Sigma_{i<j<k} a_{i} a_{j} a_{k}+\ldots .
$$

In particular,

$$
\mathrm{P}\left(a_{0}, a_{1}, 0,0, \ldots\right)=a_{0}+a_{1}+a_{0} a_{1} .
$$

Proposition 3.3. Any commutative monoid $A$ in the monoidal category SerMn has a series monoid structure defined by $0 \in A$ and $\mathrm{P}: A^{\mathbb{N}} \rightarrow A$.

Remark 3.4. Notice that each of Examples 2.4 and 2.5 can be obtained using Proposition 3.3 from an example of the countable supremum type of Example 2.7. For Example 2.4, take the sup-lattice $\mathbb{N} \cup\{\infty\}$ with addition as monoid structure. For Example 2.5, take the sup-lattice $[0, \infty]$ with addition as monoid structure. Indeed, Example 2.7 is obtained from itself using the monoid structure of finite sup. 
Remark 3.5. Notice that the unit for the monoid $A$ is not needed for Proposition 3.3. The formula (3.1) does not require commutativity of $A$ but then we only obtain a "non-commutative series monoid" in a sense to be pursued in Section 9.

Remark 3.6. As pointed out by Day [4], the ordered set $[0, \infty]$ is $*$-autonomous with multiplication as tensor product and dualizing object the same as the tensor unit 1 , internally homming into which gives reciprocal as the equivalence

$$
S:[0, \infty]^{\text {op }} \longrightarrow[0, \infty]
$$

In fact we see that $S(\alpha)=\frac{1}{\alpha}$ is actually the dual of each $0<\alpha<\infty$, while $S(0)=\infty$ and $S(\infty)=0$. Day further points out that the natural logarithm gives an inverse to a monoidal equivalence

$$
\exp :[-\infty, \infty] \longrightarrow[0, \infty]
$$

where the tensor product in the domain is addition, and therefore is *autonomous.

Motivated by Remark 3.6, we take our commutative monoid $A$ in SerMn and create another copy of the set $A$ which we will denote by $\ell A$. The elements of $\ell A$ will be denoted by $\ell a$ where $a \in A$. We make $\ell A$ into a commutative monoid by defining

$$
\ell a+\ell b=\ell(a b), 0=\ell 1 \text { and }-\infty=\ell 0 .
$$

By definition, if $a \leq 1$ in $A$ then there exists $u \in A$ with $a+u=1$. We can form the geometric series $v=1+u+u^{2}+u^{3}+\ldots$ in $A$; then $a v+u v=(a+u) v=v=1+u\left(1+u+u^{2}+\ldots\right)=1+u v$. If $u v$ can be cancelled, then $v=a^{-1}$. Then we have

$$
\ell\left(a^{-1}\right)=-\ell a
$$

We also have some countable sums in $\ell A$ :

$$
\Sigma_{n} \ell\left(1+u_{n}\right)=\Pi_{n}\left(1+u_{n}\right)=1+\mathrm{P}_{n} u_{n} .
$$

When 1 is cancellative in the additive monoid $A$, then $1 \leq a$ implies $1+u=a$ for a unique $u$; in this case, (3.6) defines a sum for sequences of "non-negative elements" $\ell a_{n}=\ell\left(1+u_{n}\right)$ in $\ell A$. 


\section{Zeno morphisms and magnitude modules}

Given an endomorphism $f: A \rightarrow A$ in SerMn, define $\tilde{f}: A \rightarrow A$ by the geometric series

$$
\tilde{f}=\Sigma_{n \in \mathbb{N}} f^{\circ(n+1)}
$$

in the pointwise structure on $A^{A}$. This $\tilde{f}: A \rightarrow A$ satisfies

$$
f \circ\left(1_{A}+\tilde{f}\right)=f+f \circ \tilde{f}=\tilde{f}
$$

and is a morphism in SerMn.

Definition 4.1. A Zeno morphism in SerMn is an endomorphism $h: A \rightarrow A$ such that $\tilde{h}=1_{A}$. A magnitude module in the sense of Higgs [9] is a series monoid equipped with a Zeno morphism $h$.

Magnitude modules are models of an algebraic theory; they are series monoids with an extra unary operation satisfying one extra axiom.

From (4.2), any Zeno morphism satisfies

$$
h+h=1_{A}
$$

and so can be regarded as the operation of halving.

Example 4.2. When $A=\mathbb{N} \cup\{\infty\}$ as in Example 2.4, there exists no Zeno morphism since (4.3) gives the contradiction $h(1)+h(1)=1$.

Example 4.3. When $A=[0, \infty]$ as in Example 2.5, the unique Zeno morphism is defined by $h(a)=\frac{1}{2} a$ and $h(\infty)=\infty$.

Example 4.4. Refer back to Example 2.6 for any set $X$ and any magnitude module $A$. The pointwise Zeno morphism makes both $A^{X}$ and $[X, A]$ into magnitude modules. For $X$ a measurable space, Higgs [9] observed that the measurable functions $f: X \rightarrow[0, \infty]$ form a magnitude submodule of $[0, \infty]^{X}$ and that magnitude module morphisms from there into $[0, \infty]$ are the countably-additive $[0, \infty]$-valued integrals on $X$.

Example 4.5. For a partially ordered set $A$ as in Example 2.7, the identity function $h(a)=a$ is Zeno.

Theorem 4.6 (Higgs). The free magnitude module on a single generating element is $[0, \infty]$ as in Example 4.3. 
Proof. The proof is given in Section 4 of [9] so we shall only give an indication. Every natural number is a finite sum $1+1+\cdots+1$ while $\infty=1+1+\ldots$. Every positive real is the sum of a natural number and a real number $t$ in the interval $[0,1)$. However, we have the binary expansion $t=\Sigma_{n \in \mathbb{N}} h^{\circ m_{n}}(1)$, where $m_{0}, m_{1}, \ldots$ is a sequence of strictly positive integers. Consequently, $1 \in[0, \infty]$ generates. The fact that we have equality of binary expansions such as $1.000 \cdots=0.111 \ldots$ is no problem since $1=\tilde{h}(1)$.

Remark 4.7. The construct of the extended reals $[0, \infty]$ as a quotient of a free series monoid is as follows. Let $\mathbb{M}=(\mathbb{N} \cup\{\infty\})^{\mathbb{N}}$ be the free series monoid on $\mathbb{N}$ (Proposition 2.14). We have the universal morphism $\chi: \mathbb{N} \rightarrow \mathbb{M}$ taking $n$ to the function $\chi_{n}$ which has only non-zero value at $n$ and that value is 1 . Take the smallest series monoid congruence $\sim$ including the relations

$$
\chi_{n} \sim \chi_{n+1}+\chi_{n+2}+\chi_{n+3}+\ldots .
$$

A consequence of these relations is $\chi_{n} \sim 2 \chi_{n+1}$. Now $\mathbb{M} / \sim$ becomes a magnitude module by the Zeno function $h$ defined by $h\left(\chi_{n}\right)=\chi_{n+1}$, that is, $h$ is induced by successor on $\mathbb{N}$. We have the magnitude module isomorphism

$$
\mathbb{M} / \sim \stackrel{\cong}{\rightarrow}[0, \infty], \quad \chi_{n} \mapsto \frac{1}{2^{n}} .
$$

Incidentally, another way of constructing the reals from endomorphisms of $\mathbb{N}$ is explained in [25]. The question of how to define multiplication for any construction of the real number system is always of interest. In [25], it is simply induced by composition of functions. For decimals, it is tricky. We now turn to the multiplication in our context.

For any series monoid $A$, by freeness there is a magnitude module morphism

$$
\lambda:[0, \infty] \longrightarrow \operatorname{ser}(A, A)
$$

taking the generator $1 \in[0, \infty]$ to the identity function of $A$; see Section 3 . This gives an action

$$
\text { : : }[0, \infty] \otimes A \longrightarrow A
$$

of $[0, \infty]$ on $A$ defined by $\alpha \cdot a=\lambda(\alpha)(a)$. In particular, we have a monoid structure

$$
\text { : : }[0, \infty] \otimes[0, \infty] \longrightarrow[0, \infty]
$$

on $[0, \infty]$ in the monoidal category SerMn; the unit is the generator 1 of $[0, \infty]$. This gives a monad $[0, \infty] \otimes-$ on SerMn.

Proposition 4.8 (Higgs). The Eilenberg-Moore algebras for the monad $[0, \infty] \otimes-$ on SerMn are precisely the magnitude modules. 
The "magnitude" terminology comes from Huntingdon [11] who took magnitudes in the unextended strictly positive reals $(0, \infty)$.

Remark 4.9. There is also what one might call the paradoxical positive reals where the geometric series $\frac{1}{2}+\frac{1}{4}+\frac{1}{8}+\ldots$ is not 1 . It is an example of a very general simple construction of an additive monoid structure, on the disjoint union $\{0\}+X+S$, from any semigroup morphism $k: S \rightarrow X$ in Set. It freely adds the zero element 0 to the semigroup $X+S$ whose addition $\mu_{X+S}$ is the composite

$$
\begin{array}{r}
(X+S) \times(X+S) \stackrel{\cong}{\rightarrow} X \times X+X \times S+S \times X+S \times S \stackrel{1+1 \times k+k \times 1+1}{\longrightarrow} \\
X \times X+X \times X+X \times X+S \times S \stackrel{\left[\mu_{X}, \mu_{X}, \mu_{X}, \mu_{S}\right]}{\longrightarrow} X+S .
\end{array}
$$

For our particular example, let $X$ be the additive semigroup of positive real numbers, presented as infinite binary expansions excluding those having only finitely many terms equal to 1 . Let $S$ be the set of all positive rational numbers of the form $\frac{m}{2^{n}}$, where $\mathrm{n}$ and $\mathrm{m}$ are integers, presented as binary expansions $s$ having only finitely many terms equal to 1 . Define $k: S \rightarrow X$ to replace the last 1 in $s$ with a 0 and all the later 0 s by 1 s; for example, $k(1.00 \ldots)=0.11 \ldots$ Then $\{0\}+X+S$ is our monoid $\mathrm{ZP}[0, \infty)$ of paradoxical positive reals. In there we have, for example,

$$
(0.11 \ldots)+(0.11 \ldots)=(0.11 \ldots)+(1.00 \ldots)=1.11 \ldots
$$

and

$$
(1.00 \ldots)+(1.00 \ldots)=10.00 \ldots .
$$

We note that $\mathrm{ZP}[0, \infty)$ is not only an ordered monoid, but, considered as an ordered set with $\infty$ added, is the free completion of $S$ under arbitrary joins; this is in contrast to the ordinary $[0, \infty]$, which is the existing-join-preserving completion.

\section{Series monoidal categories}

Let $\mathscr{A}$ be a category. Given an object 0 of $\mathscr{A}$, define the functor

$$
\delta: \mathscr{A} \longrightarrow \mathscr{A}^{\mathbb{N} \times \mathbb{N}}
$$

by

$$
\delta(A)_{m, n}= \begin{cases}A & \text { if } m=n \\ 0 & \text { if } m \neq n\end{cases}
$$


For $A \in \mathscr{A}^{\mathbb{N}}$ and a functor $\Sigma: \mathscr{A}^{\mathbb{N}} \rightarrow \mathscr{A}$, note that $\delta\left(\Sigma_{n} A_{n}\right)$ and $\Sigma_{n} \delta\left(A_{n}\right)$ are not too different:

$$
\delta\left(\Sigma_{n} A_{n}\right)_{r, s}= \begin{cases}\Sigma_{n} A_{n} & \text { if } r=s \\ 0 & \text { if } r \neq s\end{cases}
$$

while

$$
\Sigma_{n} \delta\left(A_{n}\right)_{r, s}= \begin{cases}\Sigma_{n} A_{n} & \text { if } r=s \\ \Sigma(0,0, \ldots) & \text { if } r \neq s\end{cases}
$$

So, if we have an isomorphism $\lambda_{0} 0: 0 \rightarrow \Sigma(0,0, \ldots)$, then there is an induced isomorphism $\overline{\lambda_{0} 0}: \delta\left(\Sigma_{n} A_{n}\right) \rightarrow \Sigma_{n} \delta\left(A_{n}\right)$ which is the identity on the diagonal and $\lambda_{0} 0$ elsewhere.

Definition 5.1. A series monoidal category is a category $\mathscr{A}$ equipped with an object $0 \in \mathscr{A}$, a functor

$$
\Sigma: \mathscr{A}^{\mathbb{N}} \longrightarrow \mathscr{A},\left(A_{i}\right)_{i \in \mathbb{N}} \longmapsto \Sigma_{i \in \mathbb{N}} A_{i}
$$

and natural isomorphisms
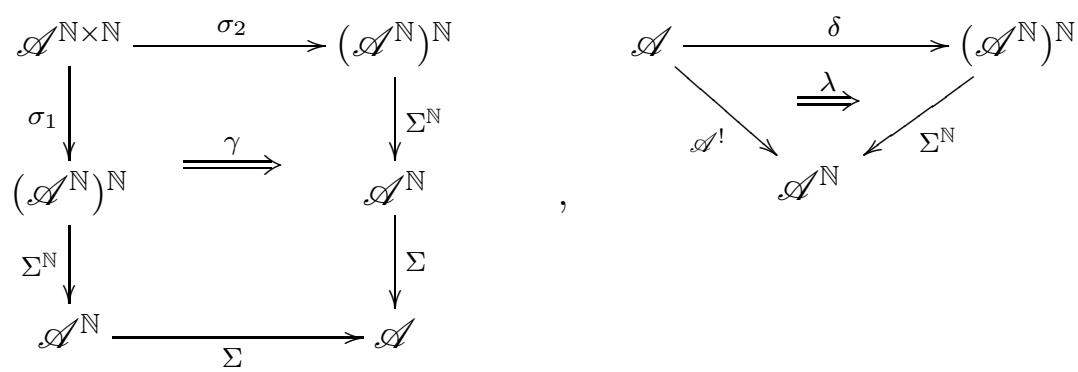

subject to the conditions that the components of the $\lambda_{n}$ at 0 are all equal and diagrams (5.2) and (5.3) commute.

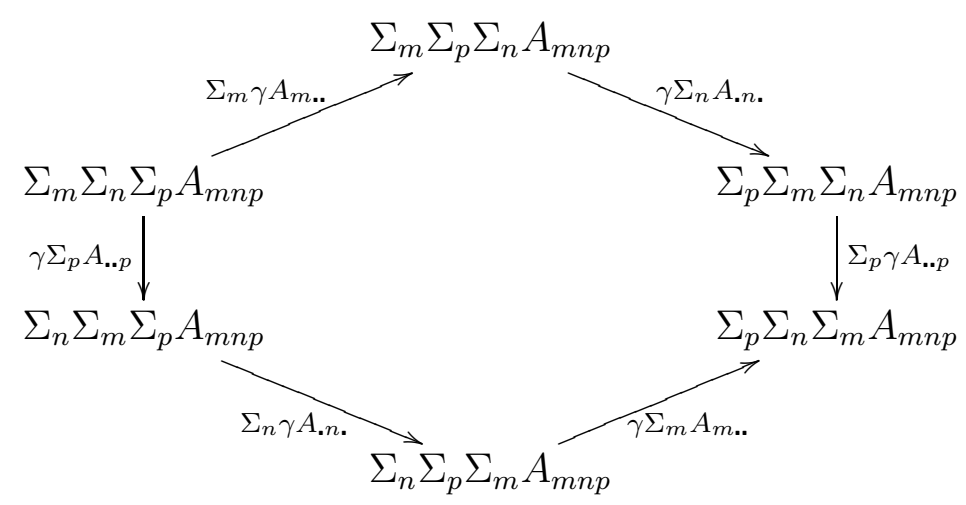




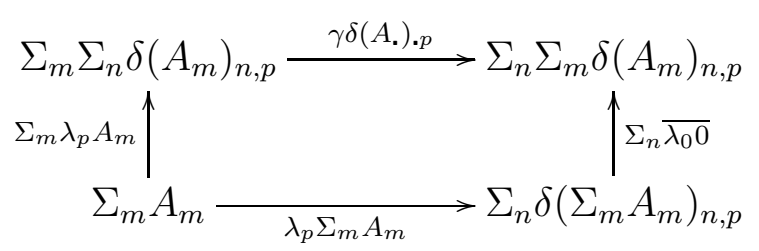

Just as Proposition 2.8 used (2.4) and (2.1), we can use (5.1) to obtain a canonical isomorphism

$$
\widehat{\xi}: \Sigma_{r} A_{\xi(r)} \cong \Sigma_{n} A_{n}
$$

for any injective function $\xi: \mathbb{N} \rightarrow \mathbb{N}$ and any $A \in \mathscr{A}^{\mathbb{N}}$ with $A_{n}=0$ for $n$ not in the image of $\xi$.

If $\xi: \mathbb{N} \rightarrow \mathbb{N} \times \mathbb{N}$ is an injective function and $A_{m, n}=0$ for $(m, n)$ not in the image of $\xi$, then we have a canonical isomorphism

$$
\widehat{\xi}: \Sigma_{r} A_{\xi(r)} \cong \Sigma_{m} \Sigma_{n} A_{(m, n)} .
$$

Clearly the dual $\mathscr{A}^{\text {op }}$ of a series monoidal category $\mathscr{A}$ is series monoidal with the same $\Sigma$ and 0 .

Example 5.2. Any category $\mathscr{A}$ with countable coproducts is series monoidal with $\Sigma$ taken to be the coproduct. Dually, any category with countable products is series monoidal. For $\mathscr{A}=$ SerMn, these two series monoidal structures coincide (by Proposition 2.13).

Example 5.3. Of course every partially ordered set is a category with at most one morphism between one object and another. This category structure is compatible with the series monoid structure of the countable-sup-lattice example (Example 2.7) and so gives a series monoidal category. This is actually a special case of Example 5.2.

Example 5.4. Indeed, every series monoid $A$ is a series monoidal category by regarding it as a discrete category. Also $A$ is a series monoidal category by regarding it as a category using the pre-order defined in Section 2; Higgs [9] proves this is a partial order when $A$ is a magnitude module.

Example 5.5. This is not an example, although claimed to be so in the published version of this paper. Let $R$ be a commutative ring and consider the category $\operatorname{Mod}_{R}$ of $R$-modules. For $A \in \operatorname{Mod}_{R}^{\mathbb{N}}$ and any $R$-module $B$, define a function $f: \prod_{n \in \mathrm{N}} A_{n} \rightarrow B$ to be multilinear when each

$$
f\left(a_{0}, a_{1}, \ldots, a_{m-1},-, a_{m+1}, \ldots\right): A_{m} \rightarrow B
$$

is an $R$-module morphism. The representing countable tensor does not give a series monoidal structure. This multiple tensor is studied in Chevalley's book [Fundamental Concepts of Algebra (Academic Press, 1956)]. For $R=\mathbb{C}, \mathrm{Ng}$ [21] makes some use of this tensor product, along with some variants. 
Example 5.6. This is not an example, although claimed to be so in the published version of this paper. For a commutative monoid $R$ in the monoidal category SerMn (Section 3), we purported to have a multilinear-style series monoidal structure on the category $\operatorname{SerMd}_{R}$ of $R$-modules, and, in particular, on SerMn. We do not.

Example 5.7. For a sequence $A=\left(A_{n}\right)_{n \in \mathbb{N}}$ of small categories and a category $X$, a funny functor $f: A \rightarrow X$ is a function assigning to each object $a \in \prod_{n} A_{n}$ an object $f(a) \in X$, equipped with the structure of a functor $A_{m} \rightarrow X$ on each object assignment $a_{m} \mapsto f(a)$ with all $a_{n} \in A_{n}$ fixed for $n \neq m$. There is a category $\ddot{\cup}_{A}=\ddot{\cup}_{n \in \mathbb{N}} A_{n}$ such that funny functors $A \rightarrow X$ are in natural bijection with functors $\ddot{\cup} A \rightarrow X$. There is a series monoidal structure on the category Cat of small categories where $\Sigma=\ddot{*}$.

We now make the natural definition of series monoidal functor.

Definition 5.8. Suppose $\mathscr{A}$ and $\mathscr{X}$ are series monoidal categories. A functor $F: \mathscr{A} \rightarrow \mathscr{X}$ is series monoidal when it is equipped with a morphism $\phi_{0}: 0 \rightarrow$ $F 0$ in $\mathscr{X}$ and a natural transformation with components

$$
\phi A: \Sigma_{n} F A_{n} \longrightarrow F \Sigma_{n} A_{n}
$$

such that diagrams (5.5) and (5.6) commute. We call $F$ series strong monoidal when $\phi$ and $\phi_{0}$ are invertible.

A series monoidal functor $M: 1 \rightarrow \mathscr{A}$ is called a series monoid in $\mathscr{A}$; that is, $M$ is an object of $\mathscr{A}$ equipped with morphisms $s_{0}: 0 \rightarrow M$ and $s: \mathbb{N} \cdot M=\Sigma(M, M, \ldots) \rightarrow M$ subject to the two conditions (5.5) and (5.6) with $\phi_{0}=s_{0}$ and $\phi *=s$ for $* \in 1$. Since series monoidal functors compose, they take series monoids to series monoids.

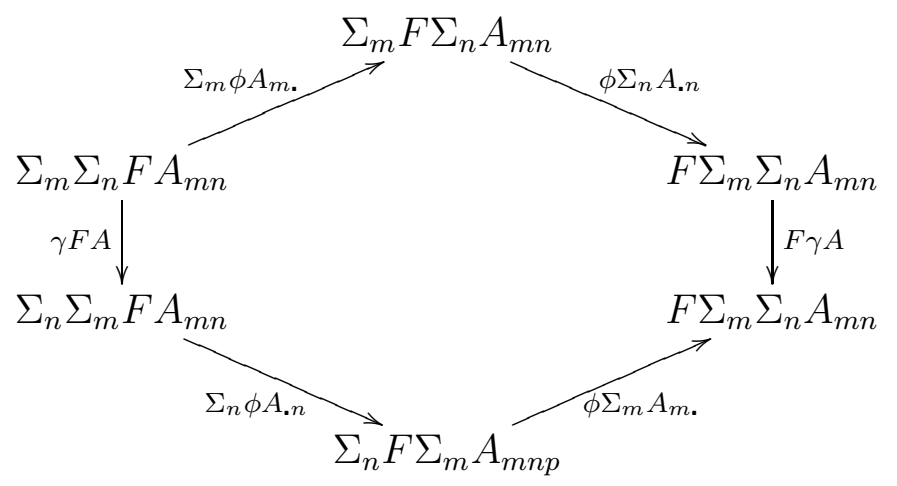




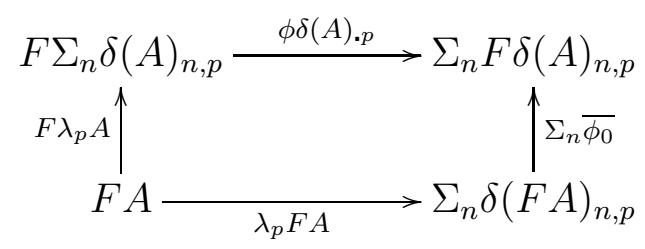

Example 5.9. For any series monoidal category $\mathscr{A}$, the hom functor

$$
\mathscr{A}(-.-): \mathscr{A}^{\mathrm{op}} \times \mathscr{A} \longrightarrow \text { Set }
$$

is series monoidal where the series monoidal structure on Set is countable product. Here $\phi_{0}: \mathbf{1} \rightarrow \mathscr{A}(0,0)$ picks out the identity morphism of $0 \in \mathscr{A}$ while $\phi(C, A)$ is the effect

$$
\prod_{n \in \mathbb{N}} \mathscr{A}\left(C_{n}, A_{n}\right)=\mathscr{A}^{\mathbb{N}}(C, A) \stackrel{\Sigma}{\rightarrow} \mathscr{A}\left(\Sigma_{n \in \mathbb{N}} C_{n}, \Sigma_{n \in \mathbb{N}} A_{n}\right)
$$

of the functor $\Sigma$ on homs. It follows that, if $C$ is a series comonoid in $\mathscr{A}$ (= series monoid in $\mathscr{A}^{\mathrm{op}}$ ) and $A$ is a series monoid in $\mathscr{A}$ (so that $(C, A$ ) is a series monoid in $\left.\mathscr{A}^{\mathrm{op}} \times \mathscr{A}\right)$, then $\mathscr{A}(C, A)$ becomes a series monoid in Set; naturally this is called convolution.

Definition 5.10. Suppose $F, G: \mathscr{A} \rightarrow \mathscr{X}$ are series monoidal functors. A natural transformation $\sigma: F \Rightarrow G$ is series monoidal when the two diagrams (5.7) commute.
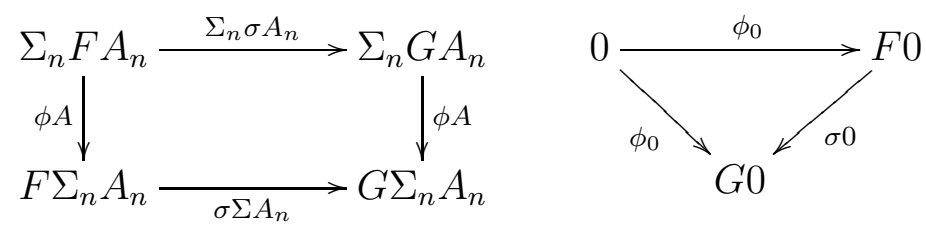

With the obvious compositions, this defines a 2-category SerMnCat. Write $\mathrm{SerMn}_{\mathrm{s}}$ Cat for the sub-2-category obtained by restricting to the series strong monoidal functors. The 2-category SerMnCat has products preserved by the forgetful 2-functor into Cat. It is immediate from the definitions that:

Proposition 5.11. For any series monoidal category $\mathscr{A}$, the functor

$$
\Sigma: \mathscr{A}^{\mathbb{N}} \rightarrow \mathscr{A}
$$

is series strong monoidal.

Associated with this kind of "commutativity" of the theory is the fact that any countable product of series monoidal categories is also the bicategorical coproduct in $\mathrm{SerMn}_{\mathrm{s}}$ Cat; that is, $\mathrm{SerMn}_{\mathrm{s}}$ Cat has countable direct sums in the bicategorical sense. 
Example 5.12. As this involved the non-Example 5.5, it was in error.

Example 5.13. As this involved the non-Example 5.6, it was also in error.

For any series monoidal category $\mathscr{A}$ and subset $S \subseteq \mathbb{N}$, we can define a functor $\Sigma_{S}: \mathscr{A}^{S} \rightarrow \mathscr{A}$ whose value at $A \in \mathscr{A}^{S}$ is

$$
\Sigma_{n \in S} A_{n}=\Sigma_{n \in \mathbb{N}} C_{n}
$$

where

$$
C_{n}= \begin{cases}A_{n} & \text { if } n \in S \\ 0 & \text { otherwise }\end{cases}
$$

When $A_{n}=B$ for all $n \in S$, we also put

$$
S \cdot B:=\Sigma_{n \in S} A_{n} .
$$

Using (5.4), we obtain, for any bijection $\xi: S \rightarrow T$, an isomorphism

$$
\widehat{\xi}: \Sigma_{r \in S} A_{\xi(r)} \cong \Sigma_{n \in T} A_{n}
$$

with the special case

$$
\widehat{\xi}: S \cdot B \cong T \cdot B
$$

This definition transports to any bijection $\xi$ between any countable sets $S$ and $T$ yielding a functor

$$
-\cdot-: \mathrm{CF} \times \mathscr{A} \longrightarrow \mathscr{A},(S, B) \mapsto S \cdot B
$$

where $\mathrm{CF}$ is the category of countable sets and all functions. Notice that, for $S, T \subseteq \mathbb{N}$ and $A=\left(A_{m, n}\right)_{(m, n) \in S \times T}$, the isomorphism $\gamma$ of (5.1) restricts to an isomorphism

$$
\gamma: \Sigma_{m \in S} \Sigma_{n \in T} A_{m, n} \cong \Sigma_{n \in T} \Sigma_{m \in S} A_{m, n} .
$$

When $S$ is any countable set and $A=\left(A_{m}\right)_{m \in T}$, this transports to an isomorphism

$$
\gamma: S \cdot \Sigma_{n \in T} A_{n} \cong \Sigma_{n \in T} S \cdot A_{n} .
$$

For any countable $T$ and $A \in \mathscr{A}$, we obtain

$$
\gamma: S \cdot(T \cdot A) \cong T \cdot(S \cdot A)
$$

and this is isomorphic to $(S \times T) \cdot A$.

Write $\mathrm{ev}_{S}: S \cdot A^{S} \rightarrow A$ for the morphism corresponding to the identity of $A^{S}$. Then each bijection $\xi: S \rightarrow T$ determines an isomorphism

$$
\bar{\xi}: A^{S} \rightarrow A^{T}
$$

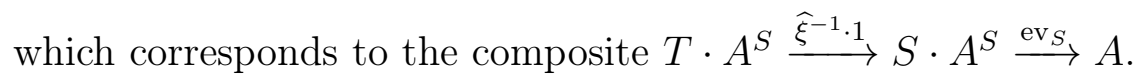


Proposition 5.14. The tensor product defined by

$$
A_{1}+A_{2}=\Sigma_{n \in\{1,2\}} A_{n} .
$$

renders $\mathscr{A}$ symmetric monoidal with 0 as tensor unit. Moreover, $\Sigma: \mathscr{A}^{\mathbb{N}} \rightarrow \mathscr{A}$ is a symmetric strong monoidal functor.

For series monoidal categories $\mathscr{A}$ and $\mathscr{X}$, the category $\operatorname{SerMn}_{\mathrm{s}} \operatorname{Cat}(\mathscr{A}, \mathscr{X})$ is series monoidal under the pointwise series monoidal structure; we write $\operatorname{Ser}(\mathscr{A}, \mathscr{X})$ for this series monoidal category. For a sequence $F=\left(F_{n}\right)_{n \in \mathbb{N}}$ of series strong monoidal functors $F_{n}: \mathscr{A} \rightarrow \mathscr{X}$, the definition of $\Sigma F$ is the composite

$$
\mathscr{A} \stackrel{\left(F_{n}\right)}{\rightarrow} \mathscr{X}^{\mathbb{N}} \stackrel{\Sigma}{\rightarrow} \mathscr{X}
$$

The forgetful 2-functor

$$
\mathrm{U}: \mathrm{SerMn}_{\mathrm{s}} \mathrm{Cat} \rightarrow \mathrm{Cat}
$$

is monadic in a bicategorical sense. In particular, it has a left biadjoint (see [3] for this sort of result) whose value at the terminal category can be made explicit.

Proposition 5.15. The 2-functor (5.17) is pseudo-representable by the series monoidal category $\mathrm{CB}$ with countable sets as objects, bijective functions as morphisms, and disjoint union as $\Sigma$. To be precise, for any series monoidal category $\mathscr{A}$, the category of series strong monoidal functors $\mathrm{CB} \rightarrow \mathscr{A}$ is pseudonaturally equivalent to the category $\mathscr{A}$. A series monoidal equivalence

$$
\operatorname{Ser}(\mathrm{CB}, \mathscr{A}) \simeq \mathscr{A}
$$

defined by evaluation at the singleton set, follows therefrom.

Proof. Given an object $A \in \mathscr{A}$, we define a series strong monoidal functor $F: \mathrm{CB} \rightarrow \mathscr{A}$ with $F \mathbf{1}=A$ as follows. Define $F-=-\cdot A$ as per (5.12) with series monoidal structure supplied by (5.13). The assignment $A \mapsto F$ is the object function for a functor $\mathscr{A} \rightarrow \operatorname{Ser}(\mathrm{CB}, \mathscr{A})$ defined on morphisms by universality. This provides the inverse equivalence to evaluation at $\mathbf{1}$.

We also have the 2-functor

$$
\text { sermn: } \operatorname{SerMn}_{\mathrm{s}} \mathrm{Cat} \longrightarrow \text { Cat }
$$

which takes each series monoidal category $\mathscr{A}$ to the category

$$
\operatorname{sermn} \mathscr{A}=\operatorname{SerMnCat}(\mathbf{1}, \mathscr{A})
$$

of series monoids in $\mathscr{A}$. 
Proposition 5.16. The 2-functor (5.18) is pseudo-representable by the series monoidal category $\mathrm{CF}$ with countable sets as objects, functions as morphisms, and disjoint union (coproduct) as $\Sigma$. To be precise, an equivalence of categories

$$
\operatorname{SerMn}_{\mathrm{s}} \operatorname{Cat}(\mathrm{CF}, \mathscr{A}) \simeq \operatorname{sermn} \mathscr{A},
$$

pseudonatural in series monoidal categories $\mathscr{A}$.

Proof. Given a series monoid $A \in \mathscr{A}$, we have the series strong monoidal functor $F: \mathrm{CB} \rightarrow \mathscr{A}$ with $F \mathbf{1}=A$ as in Proposition 5.15. Using the series monoid structure $s_{0}: 1 \rightarrow A, s: \mathbb{N} \cdot A \rightarrow A$ on $A$, we can extend $F$ to a series strong monoidal functor $F^{\prime}: \mathrm{CF} \rightarrow \mathscr{A}$ as follows. For any $S \subseteq \mathbb{N}$, let $A_{n}=A$ for all $n \in \mathbb{N}$, let $C_{n}=A$ for all $n \in S$, let $C_{n}=1$ for all $n \notin S$, and let $u_{n}: C_{n} \rightarrow A_{n}$ be the identity of $A$ for $n \in S$ and $s_{0}$ otherwise. We can define

$$
\left(S \cdot A \stackrel{s_{S}}{\longrightarrow} A\right)=\left(\Sigma_{n} C_{n} \stackrel{\Sigma_{n} u_{n}}{\longrightarrow} \Sigma_{n} A_{n} \stackrel{s}{\rightarrow} A\right) .
$$

For any order-preserving function $\alpha: S \rightarrow T$ between subsets of $\mathbb{N}$, we obtain

$$
\left(S \cdot A \stackrel{s_{\alpha}}{\longrightarrow} T \cdot A\right):=\left(\Sigma_{n \in T} \alpha^{-1}(n) \cdot A \stackrel{\Sigma_{n \in T} s_{\alpha}-1(n)}{\longrightarrow} \Sigma_{n \in T} A\right) .
$$

For a bijective $\xi: S \rightarrow S$, we already have $\widehat{\xi}: S \cdot A \cong S \cdot A$ as in (5.11). As every function $\alpha: S \rightarrow T$ is a composite of an automorphism $\alpha_{1}: S \rightarrow S$ and an order-preserving function $\alpha_{2}: S \rightarrow T$, we obtain

$$
F \alpha=s_{\alpha}=s_{\alpha_{2}} \circ \widehat{\alpha_{1}}: S \cdot A \rightarrow T \cdot A
$$

in $\mathscr{A}$. The remaining details of the proof that this gives an inverse equivalence are as for finite sets, symmetric monoidal categories, and commutative monoids.

As a bicategory, $\operatorname{SerMn}_{\mathrm{s}}$ Cat is symmetric closed monoidal in the sense of [5]. There is a tensor product $\mathscr{A} \otimes \mathscr{B}$ satisfying pseudonatural equivalences

$$
\begin{aligned}
\operatorname{SerMn}_{\mathrm{s}} \operatorname{Cat}(\mathscr{A}, \operatorname{Ser}(\mathscr{B}, \mathscr{X})) & \simeq \operatorname{Ser} \operatorname{Mn}_{\mathrm{s}} \operatorname{Cat}(\mathscr{A} \otimes \mathscr{B}, \mathscr{X}) \\
& \simeq \operatorname{Ser} \operatorname{Mn}_{\mathrm{s}} \operatorname{Cat}(\mathscr{B}, \operatorname{Ser}(\mathscr{A}, \mathscr{X})) .
\end{aligned}
$$

A diagonal for an object $A$ of a series monoidal category $\mathscr{A}$ is a morphism $d: A \longrightarrow \Sigma(A, A, \ldots)$ such that, for all bijections $\xi: \mathbb{N} \rightarrow \mathbb{N} \times \mathbb{N}$, the following square commutes.

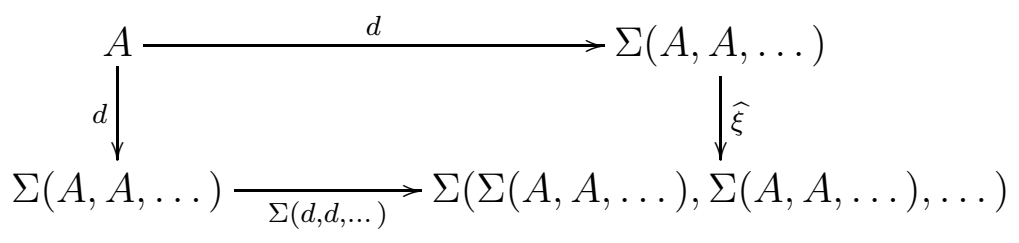


Definition 5.17. A magnitude module $M$ in a series monoidal category $\mathscr{A}$ is a series monoid equipped with a diagonal morphism $d: M \longrightarrow \Sigma(M, M, \ldots)$ and a series monoid endomorphism $h: M \rightarrow M$ such that the composite

$$
\tilde{h}: M \stackrel{d}{\rightarrow} \Sigma(M, M, \ldots) \stackrel{(h, h \circ h, h \circ h \circ h, \ldots)}{\longrightarrow} \Sigma(M, M, \ldots) \stackrel{s}{\rightarrow} M
$$

is the identity of $M$.

\section{Zeno functors and magnitude categories}

Let $F: \mathscr{A} \rightarrow \mathscr{A}$ be a series monoidal endofunctor on the series monoidal category $\mathscr{A}$. For each $n \in \mathbb{N}$, we have the $n$-fold composite series monoidal endofunctor

$$
F^{\circ n}=\overbrace{F \circ F \circ \ldots F}^{n}: \mathscr{A} \longrightarrow \mathscr{A} \text {. }
$$

By the product property of $\mathscr{A}^{\mathbb{N}}$ in SerMnCat, a series monoidal functor

$$
F^{\circ(\cdot+1)}: \mathscr{A} \longrightarrow \mathscr{A}^{\mathbb{N}}
$$

is induced. This composes with the series strong monoidal functor $\Sigma$ of Proposition 5.11 to yield a series monoidal functor

$$
\tilde{F}=\Sigma_{n \in \mathrm{N}} F^{\circ(n+1)}: \mathscr{A} \longrightarrow \mathscr{A} .
$$

There are canonical natural isomorphisms

$$
F+F \circ \tilde{F} \cong \tilde{F} \text { and } \tilde{F} \circ F \cong F \circ \tilde{F} .
$$

Definition 6.1. A Zeno functor on $\mathscr{A}$ is a series strong monoidal functor $H: \mathscr{A} \rightarrow \mathscr{A}$ equipped with a series monoidal isomorphism $\kappa: 1_{\mathscr{A}} \cong \tilde{H}$ such that (6.3) commutes. A magnitude category is a series monoidal category equipped with a Zeno functor $H$.

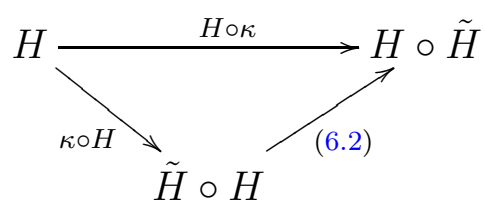

Example 6.2. When $\mathscr{A}=\mathrm{CB}$ or $\mathscr{A}=\mathrm{CF}$, there exists no Zeno functor since 1 is not isomorphic to a disjoint union of a set with itself.

Example 6.3. Any magnitude module, either as a discrete category or with its partial order, is a magnitude category. 
Example 6.4. For any category $\mathscr{C}$ and magnitude category $\mathscr{A}$, the functor category $[\mathscr{C}, \mathscr{A}]$ is a magnitude category with the pointwise structure. If $\mathscr{C}$ is serial monoidal then $\operatorname{Ser}(\mathscr{C}, \mathscr{A})$ is a magnitude subcategory of $[\mathscr{C}, \mathscr{A}]$.

Definition 6.5. A magnitude functor $F: \mathscr{A} \rightarrow \mathscr{X}$ is a series monoidal functor equipped with a series monoidal natural transformation $\nu_{1}: H \circ F \Rightarrow$ $F \circ H$ compatible with the series monoidal isomorphisms $\kappa: 1_{\mathscr{A}} \cong \tilde{H}$ in the sense that (6.4) should commute. It is strong when it is series strong monoidal and $\nu_{1}$ is invertible.

The natural transformation $\nu_{1}: H \circ F \Rightarrow F \circ H$ inductively determines natural transformations $\nu_{n}: H^{\circ n} \circ F \Rightarrow F \circ H^{\circ n}$ via

$$
\nu_{n+1}: H^{\circ(n+1)} \circ F \stackrel{H^{\circ n} \nu_{1}}{\longrightarrow} H^{\circ n} \circ F \circ H \stackrel{\nu_{n} \circ H}{\longrightarrow} F \circ H^{\circ(n+1)},
$$

and hence a natural transformation

$$
\tilde{\nu}=\Sigma_{n>0} \nu_{n}: F \circ \tilde{H} \Rightarrow \tilde{H} \circ F .
$$

We ask commutativity of

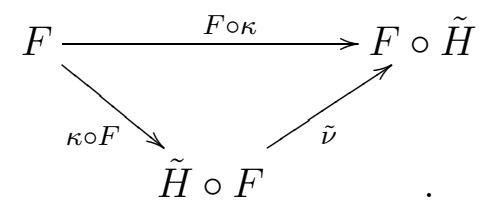

Example 6.6. For a magnitude category $\mathscr{A}$, the Zeno functor $H: \mathscr{A} \rightarrow \mathscr{A}$ is a magnitude functor with $\nu_{1}=1_{H \circ H}$.

Definition 6.7. Suppose $F, G: \mathscr{A} \rightarrow \mathscr{X}$ are magnitude functors. A magnitude natural transformation $\sigma: F \Rightarrow G$ is a series monoidal natural transformation for which the following square commutes.

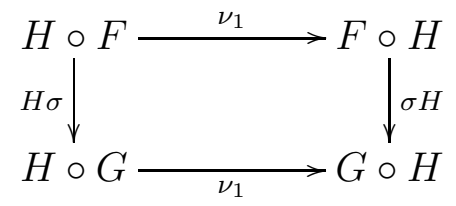

With the obvious compositions, this defines a 2-category MgnCat of magnitude categories, magnitude functors and magnitude natural transformations. We write $\operatorname{Mgn}_{\mathrm{s}}$ Cat for the sub-2-category obtained by restricting to strong magnitude functors.

If $\mathscr{C}$ and $\mathscr{A}$ are magnitude categories then the category $\operatorname{Mgn}(\mathscr{C}, \mathscr{A})$ of strong magnitude functors and magnitude natural transformations is a magnitude subcategory of $\operatorname{Ser}(\mathscr{C}, \mathscr{A})$. 
The countable direct sums of $\mathrm{SerMn}_{\mathrm{s}}$ Cat restrict to $\mathrm{Mgn}_{\mathrm{s}}$ Cat; that is, countable products restrict and the appropriate coproduct injections are magnitude functors.

The forgetful 2-functor

$$
\mathrm{U}: \mathrm{Mgn}_{\mathrm{s}} \mathrm{Cat} \rightarrow \mathrm{Cat}
$$

is monadic in the bicategorical sense. In particular, it has a left biadjoint.

Definition 6.8. The value at the terminal category of the left biadjoint to $U$ is called the magnitude groupoid of positive real sets and denoted by RSet.

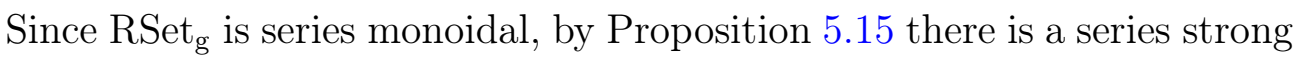
monoidal functor

$$
\mathrm{I}: \mathrm{CB} \longrightarrow \mathrm{RSet}_{\mathrm{g}}
$$

from the series monoidal category of countable sets for which $\mathrm{I}(\mathbf{1})$ is the generator of RSet ${ }_{g}$, which generator we shall also denote by 1 . Indeed, we shall put $\mathbf{n}:=\mathrm{I}(\mathbf{n})$. We conjecture that $\mathrm{I}$ is faithful.

There is also a strong magnitude functor

$$
\#: \text { RSet }_{\mathrm{g}} \longrightarrow[0, \infty]
$$

called cardinality, taking the generator 1 to the real number 1. It follows that the composite

$$
\mathrm{CB} \stackrel{\mathrm{I}}{\rightarrow} \mathrm{RSet}_{\mathrm{g}} \stackrel{\#}{\rightarrow}[0, \infty]
$$

takes each countable set to its cardinality.

For any magnitude category $\mathscr{A}$, by freeness there is a strong magnitude functor

$$
L: \operatorname{RSet}_{\mathrm{g}} \longrightarrow \operatorname{Mgn}(\mathscr{A}, \mathscr{A})
$$

taking the generator $\mathbf{1} \in \mathrm{RSet}_{\mathrm{g}}$ to the identity functor of $\mathscr{A}$; see (5.16). This gives an action

$$
\text { - : RSet } \mathrm{g} \otimes \mathscr{A} \longrightarrow \mathscr{A}
$$

of RSet on $\mathscr{A}$ defined by $S \bullet A=(L S) A$. In particular, we have a monoidal structure

$$
\text { - : } \operatorname{RSet}_{\mathrm{g}} \otimes \text { RSet }_{\mathrm{g}} \longrightarrow \text { RSet }_{\mathrm{g}}
$$

on $\mathrm{RSet}_{\mathrm{g}}$ in the monoidal bicategory $\operatorname{SerMn}_{\mathrm{s}}$ Cat; the unit is the generator $\mathbf{1}$ of RSet .

Proposition 6.9. The pseudoalgebras for the pseudomonad $\mathrm{RSet}_{\mathrm{g}} \otimes-$ on $\mathrm{SerMn}_{\mathrm{s}}$ Cat are the magnitude categories. 
We define some objects of RSet $t_{\mathrm{g}}$ by

$$
\frac{1}{2}=H(\mathbf{1}), \frac{1}{2^{n}}=H^{\circ n}(\mathbf{1}), \frac{1}{3}=\Sigma_{n>0} \frac{1}{2^{2 n}}, \frac{1}{4}=\frac{1}{2^{2}}, \frac{\mathbf{m}}{2^{2 n}}=\mathbf{m} \bullet \frac{1}{2^{2 n}},
$$

and so on. For any natural number $k$, let $t$ be the first natural number with $k \leq 2^{t}$ and can define

$$
\frac{\mathbf{1}}{\mathbf{k}}=\Sigma_{n \in \mathbb{N}}\left(\frac{\left(\mathbf{2}^{\mathbf{t}}-\mathbf{k}\right)^{n}}{\mathbf{2}^{t(n+1)}}\right)
$$

More typically, to obtain an object of cardinality $\pi$, express $\pi-3=$ 0. $a_{1} a_{2} \ldots$ in binary form, let $a_{m_{n}}=1$ be the $n$th non-zero term in that expansion. Then

$$
\Pi=3+\frac{1}{2^{m_{1}}}+\frac{1}{2^{m_{2}}}+\ldots
$$

is an object of RSet $_{\mathrm{g}}$ with $\# \Pi=\pi$. A more difficult question is whether $\Pi$ has interesting automorphisms.

For any magnitude category $\mathscr{A}$, we can define an exponential functor

$$
E: \mathscr{A} \longrightarrow \mathscr{A}
$$

by

$$
E(X)=\Sigma_{n \in \mathbb{N}} \frac{\mathbf{1}}{\mathbf{n} !} \bullet X^{\bullet n} .
$$

We also have the 2-functor

$$
\text { sermn: } \mathrm{Mgn}_{\mathrm{s}} \mathrm{Cat} \longrightarrow \text { Cat }
$$

which takes each magnitude category $\mathscr{A}$ to the category sermn $\mathscr{A}$ of series monoids in $\mathscr{A}$.

Definition 6.10. The pseudo-representing object for the 2-functor (6.8) is called the magnitude category of positive real sets and denoted by RSet. That is, there is an equivalence of categories

$$
\operatorname{Mgn}_{\mathrm{s}} \operatorname{Cat}(\text { RSet, } \mathscr{A}) \cong \operatorname{sermn} \mathscr{A},
$$

pseudonatural in magnitude categories $\mathscr{A}$.

There is a magnitude functor RSet $g \longrightarrow$ RSet taking 1 to the generator 1 of RSet. 
Remark 6.11. Here is a construction of RSet in the spirit of Remark 4.7. Begin with the pointwise series monoidal category $\mathscr{R}_{1}=\mathrm{CF}^{\mathbb{N}}$ of sequences of countable sets. We have a series strict monoidal functor $H_{1}: \mathscr{R}_{1} \rightarrow \mathscr{R}_{1}$ defined as the suspension $H_{1}\left(X_{0}, X_{1}, X_{2}, \ldots\right)=\left(0, X_{0}, X_{1}, \ldots\right)$. We then form the series strict monoidal functor $\tilde{H}_{1}$; the formula is

$$
\tilde{H}_{1}\left(X_{0}, X_{1}, \ldots\right)=\left(0, X_{0}, X_{0}+X_{1}, \ldots\right)=\left(\Sigma_{n<0} X_{n}, \Sigma_{n<1} X_{n}, \Sigma_{n<2} X_{n}, \ldots\right) \text {. }
$$

Note that $\tilde{H}_{1} \circ H_{1}=H_{1} \circ \tilde{H}_{1}$. Now form the isocoinserter (6.9) of the identity functor of $\mathscr{R}_{1}$ and $\tilde{H}_{1}$ in the 2-category $\operatorname{SerMn}_{\mathrm{s}}$ Cat.

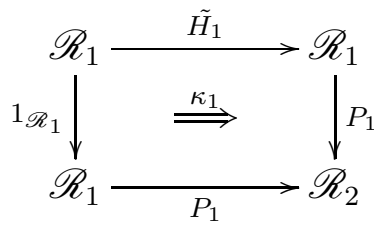

Since we have the isomorphism $P_{1} \circ H_{1} \stackrel{\kappa_{1} H_{1}}{\longrightarrow} P_{1} \circ \tilde{H}_{1} \circ H_{1}=P_{1} \circ H_{1} \circ \tilde{H}_{1}$, the universal property of (6.9) yields a unique series strong monoidal functor $H_{2}: \mathscr{R}_{2} \rightarrow \mathscr{R}_{2}$ such that $H_{2} \circ P_{1}=P_{1} \circ H_{1}$ and $H_{2} \circ \kappa_{1}=\kappa_{1} \circ H_{1}$. It follows that $\tilde{H}_{2} \circ P_{1}=P_{1} \circ \tilde{H}_{1}$ and $\tilde{H}_{2} \circ \kappa_{1}=\kappa_{1} \circ \tilde{H}_{1}$. So the universal property of (6.9) yields a unique series strong monoidal natural isomorphism $\kappa_{2}: 1_{\mathscr{R}_{2}} \Rightarrow \tilde{H}_{2}$ such that $\kappa_{2} \circ P_{1}=\kappa_{1}$. Note also that $\tilde{H}_{2} \circ H_{2}=H_{2} \circ \tilde{H}_{2}$. So we have two 2-cells $\mathrm{H}_{2} \circ \kappa_{2}$ and $\kappa_{2} \circ \mathrm{H}_{2}$ from $\mathrm{H}_{2}$ to $\tilde{H}_{2} \circ \mathrm{H}_{2}$ and we can take their coequifier $P_{2}: \mathscr{R}_{2} \rightarrow \mathscr{R}$ in the 2 -category $\mathrm{SerMn}_{\mathrm{s}}$ Cat. From the universal property of the coequifier, we obtain a unique series strong monoidal functor $H: \mathscr{R} \rightarrow \mathscr{R}$ with $P_{2} \circ H_{2}=H \circ P_{2}$, and then obtain a unique series monoidal natural isomorphism $\kappa: 1_{\mathscr{R}} \Rightarrow \tilde{H}$ with $\kappa \circ P_{2}=P_{2} \circ \kappa_{2}$. Now (6.3) is satisfied and we have a Zeno functor $(H, \kappa)$ making $\mathscr{R}$ a magnitude category.

Proposition 6.12. The magnitude category $\mathscr{R}$ constructed in Remark 6.11 is equivalent to RSet.

Proof. The universal properties of $\mathscr{R}_{2}$ and $\mathscr{R}$ combine to show that strong magnitude functors $F: \mathscr{R} \rightarrow \mathscr{A}$ are in bijection with series strong monoidal functors $G: \mathscr{R}_{1} \rightarrow \mathscr{A}$ equipped with a series monoidal isomorphism

$$
\nu_{1}: H \circ G \Rightarrow G \circ H_{1} .
$$

However, $\mathscr{R}_{1}=\mathrm{CF}^{\mathrm{N}}$ is the coproduct of countably many copies of CF. So, to give $G$ is equivalently to give a sequence of series strong monoidal functors $G_{n}: \mathrm{CF} \rightarrow \mathscr{A}$. By Proposition 5.16, to give such a sequence is equivalent to giving a sequence of series monoids $A_{n}$ in $\mathscr{A}$. However, $\nu_{1}$ induces a series monoid isomorphism $\nu_{n}: H A_{n} \cong A_{n+1}$. So the sequence of series monoids is, up to canonical isomorphism, determined by the single series monoid $A=A_{0}$. 


\section{Remarks on integer sets}

To obtain reals from integers, Higgs taught us to introduce a halving operation. It is obvious to all that to obtain integers from natural numbers, we need to introduce a minus operation. A categorical version of minus might be dual. If we think of the categorical integers as forming the free symmetric monoidal category on a single generating object, we might think of the categorical integers as forming a compact closed category in the sense of Kelly [14]; this includes symmetry.

Let symMon denote the groupoid-enriched category of symmetric monoidal categories, symmetric strong monoidal functors, and monoidal natural isomorphisms. Let CmpClsd denote the full sub-groupoid-enriched category of symMon consisting of the compact closed categories. The inclusion

$$
\text { CmpClsd } \longrightarrow \text { symMon }
$$

has a left biadjoint. The value of this biadjoint at the category FB of finite sets and bijections might be a candidate for a category ZSet of integer sets.

A category IntRel of integer sets and relations was introduced in [13]. It is the free tortile monoidal category on the symmetric traced monoidal category Rel of sets and relations. The explicit description can be found in Section 6 of [13]: the objects are pairs $(X, U)$ of sets and the morphisms $R:(X, U) \rightarrow(Y, V)$ are relations from $X+V$ to $Y+U$, while the composition uses the trace. Trace categorizes the cancellation property of addition of natural numbers.

Lemma 7.1. If a morphism $R: X+U \rightarrow Y+U$ in Rel is the graph of an injective function $f: X+U \rightarrow Y+U$ and $U$ is a finite set then the trace $\operatorname{Tr}_{X, Y}^{U}(R): X \rightarrow Y$ is the graph of an injective function $\operatorname{Tr}^{U}(f): X \rightarrow Y$. Indeed, $\operatorname{Tr}^{U}(f)(x)=f^{\circ n}(x) \in Y$ where $n>0$ is such that $f^{\circ m}(x) \in U$ for all $m<n$. If $f: X+U \rightarrow Y+U$ is bijective then so is $\operatorname{Tr}^{U}(f): X \rightarrow Y$.

Proof. This is an easy exercise for a reader who recalls the matrix formula for $\operatorname{Tr}_{X, Y}^{U}(R)$ in [13]. The reason there is such an $n$ is that $U$ is finite.

Therefore, we may wish to replace symMon by the groupoid-enriched category symMon $_{*}$ of symmetric monoidal categories for which the tensor unit is initial. Then the appropriate replacement for FB is the category FI of finite sets and injective functions; by Lemma 7.1, both of these are traced monoidal subcategories of Rel.

This produces two candidates IntFI and IntFB for categories of integer sets. The objects are pairs $(X, U)$ of finite sets and the morphisms 
$f:(X, U) \rightarrow(Y, V)$ are injective or bijective functions $f: X+V \rightarrow Y+U$, respectively. The composite $g \circ f$ of $f:(X, U) \rightarrow(Y, V)$ and $g:(Y, V) \rightarrow$ $(Z, W)$ is the trace $\operatorname{Tr}^{V}(g \# f)$ of the function $g \# f: X+W+V \rightarrow Z+U+V$ defined as follows. For $p \in X+V$,

$$
g \# f(p)= \begin{cases}g(f(p)) \in Z+V & \text { if } f(p) \in Y \\ f(p) \in U & \text { otherwise }\end{cases}
$$

while $g \# f(p)=g(p) \in Z+V$ for $p \in W$.

Of course, $\mathbb{N}$ is traced monoidal as an ordered set under addition. We have the inclusion functor $\mathrm{FB} \rightarrow \mathrm{FI}$ and the cardinality functor $\mathrm{FI} \rightarrow \mathbb{N}$ which are both traced symmetric strong monoidal. Since Int is a groupoid-enriched functor, we obtain symmetric strong monoidal functors

$$
\operatorname{IntFB} \longrightarrow \operatorname{IntFI} \longrightarrow \mathbb{Z}
$$

providing cardinalities for "integer sets".

A geometric approach, based on the idea that Euler characteristic extends cardinality, is that of Schanuel [22].

Remark 7.2. There is a classical obstruction to having both associative infinite sums and negatives. The only element $c$ with an inverse $-c$ for the binary addition in a series monoid is 0 . The proof goes back to Euler:

$0=0+0+\cdots=(c-c)+(c-c)+\cdots=c+(-c+c)+(-c+c)+\cdots=c$.

A different tack, suggested by Remark 3.6 following Day, is to note that RSet $_{\mathrm{g}}$ itself with the multiplication monoidal structure (6.5), might be considered up to equivalence to be not just positive but all extended real sets, with addition as the monoidal structure. We do not know whether this structure is $*$-autonomous.

\section{Categories enriched in a series monoidal category}

Let $\mathscr{V}$ denote a series monoidal category. It becomes symmetric monoidal under binary summation according to Proposition 5.14. The usual notion of $\mathscr{V}$-category $\mathscr{A}$ makes sense as per [16]. Because of the symmetry, the opposite $\mathscr{A}^{\text {op }}$ and tensor product, here written as a sum $\mathscr{A}+\mathscr{B}$, of enriched categories are already defined. 
What we wish to point out now is the possibility to sum series of $\mathscr{V}$ categories. By Proposition 5.14, we have the symmetric strong monoidal functor $\Sigma: \mathscr{V}^{\mathbb{N}} \rightarrow \mathscr{V}$ and so, the symmetric strong monoidal 2-functor

$$
\Sigma_{*}: \mathscr{V}^{\mathbb{N}}-\mathrm{Cat} \longrightarrow \mathscr{V} \text {-Cat }
$$

in the notation of Eilenberg-Kelly [6]. There is also the symmetric strong monoidal 2-functor

$$
\mathrm{Q}:(\mathscr{V} \text {-Cat })^{\mathbb{N}} \longrightarrow \mathscr{V}^{\mathbb{N}} \text {-Cat }
$$

taking a sequence $\mathscr{A}=\left(\mathscr{A}_{n}\right)_{n \in \mathbb{N}}$ of $\mathscr{V}$-categories to the $\mathscr{V}^{\mathbb{N}}$-category Q $\mathscr{A}$ whose objects are objects of the cartesian product $\prod_{n \in \mathbb{N}} \mathscr{A}_{n}$ and whose homs are defined by $\mathrm{Q} \mathscr{A}(A, B)_{n}=\mathscr{A}_{n}\left(A_{n}, B_{n}\right)$. Now define $\Sigma$ by composing thus:

$$
\Sigma:(\mathscr{V} \text {-Cat })^{\mathbb{N}} \stackrel{\mathrm{Q}}{\rightarrow} \mathscr{V}^{\mathbb{N}} \text {-Cat } \stackrel{\Sigma_{*}}{\rightarrow} \mathscr{V} \text {-Cat . }
$$

Explicitly, for a sequence $\mathscr{A}=\left(\mathscr{A}_{n}\right)_{n \in \mathbb{N}}$ of $\mathscr{V}$-categories, the objects of $\Sigma \mathscr{A}$ are families $A=\left(A_{n}\right)_{n \in \mathbb{N}}$ of objects $A_{n} \in \mathscr{A}_{n}$, whereas the homs are defined by $\Sigma \mathscr{A}(A, B)=\Sigma_{n} \mathscr{A}_{n}\left(A_{n}, B_{n}\right)$. In the obvious sense:

Proposition 8.1. The 2-category $\mathscr{V}$-Cat is series monoidal with this choice of $\Sigma$.

Proposition 8.2. There is a series monoidal 2-functor

$$
\text { SerMnCat } \rightarrow \text { sMon2-Cat }
$$

taking $\mathscr{V}$ to $\mathscr{V}$-Cat and $F: \mathscr{V} \rightarrow \mathscr{W}$ to $F_{*}: \mathscr{V}$-Cat $\rightarrow \mathscr{W}$-Cat.

When our base series monoidal category $\mathscr{V}$ is cocomplete in a manner allowing discussion of the bicategory $\mathscr{V}$-Mod of $\mathscr{V}$-categories and $\mathscr{V}$-modules, the operation (8.1) extends to

$$
\Sigma:(\mathscr{V}-\operatorname{Mod})^{\mathbb{N}} \longrightarrow \mathscr{V}-\operatorname{Mod}
$$

This provides an example $\mathscr{V}$-Mod of a series monoidal bicategory, leading on to series promonoidal categories and convolution.

\section{$9 \omega$-Magmas and $\omega$-monoids}

It is natural to define monoidal categories before symmetric monoidal categories, yet here, with the countable version, we have presented the commutative case without mentioning the non-commutative possibility. The next two sections correct that omission for posterity. 
Now we wish to pass to multiplicative terminology rather than additive. We call a series magma $A$ an $\omega$-magma when the operation $\Sigma: A^{\mathbb{N}} \rightarrow A$ is denoted by $\otimes: A^{\omega} \rightarrow A$, where $\omega=\mathbb{N}$ as a linearly ordered set, and $0 \in A$ is denoted by $1 \in A$. The informal notation $\otimes_{n \in \omega} a_{n}=a_{0} \otimes a_{1} \otimes \ldots$ is also helpful.

We recall (2.2) in the new notation. For any $\omega$-magma $A$ and subset $S \subseteq \omega$, we can define an operation $\otimes_{S}: A^{S} \rightarrow A$ whose value at $a \in A^{S}$ is

$$
\otimes_{n \in S} a_{n}=\otimes_{n \in \omega} c_{n}
$$

where

$$
c_{n}= \begin{cases}a_{n} & \text { if } n \in S \\ 1 & \text { otherwise }\end{cases}
$$

Definition 9.1. An $\omega$-monoid $A$ is an $\omega$-magma such that, for all orderpreserving functions $\xi: \omega \rightarrow \omega$,

$$
\otimes_{n \in \omega} \otimes_{m \in \xi^{-1}(n)} a_{m}=\otimes_{n \in \omega} a_{n}
$$

Remark 9.2. Each fibre $\xi^{-1}(n)$ of an order-preserving function $\xi: \omega \rightarrow \omega$ is either finite or forms a final segment of $\omega$. The latter case occurs only if $\xi$ has finite image, and then only for the last fibre.

Remark 9.3. After submitting the present paper, it came to our notice that (9.2) is also the "general associativity postulate" (II') of Tarski [29]. He claimed it too restrictive for his purposes.

Example 9.4. Every series monoid is an $\omega$-monoid with $\otimes=\Sigma$ and $1=0$.

Example 9.5. If $A$ admits an associative binary multiplication in SerMn then $A$ becomes an $\omega$-monoid with $\otimes=\mathrm{P}$ (see (3.1)) and $1=0$. This was foreshadowed in Remark 3.5.

\section{$10 \omega$-Monoidal categories}

For any category $\mathscr{A}$, object $I \in \mathscr{A}$, and functor

$$
\otimes: \mathscr{A}^{\omega} \longrightarrow \mathscr{A},\left(A_{i}\right)_{i \in \omega} \longmapsto \otimes_{i \in \omega} A_{i}
$$

each subset $S \subseteq \omega$, determines an operation $\otimes_{S}: \mathscr{A}^{S} \rightarrow \mathscr{A}$ whose value at $A \in \mathscr{A}^{S}$ is

$$
\otimes_{n \in S} A_{n}=\otimes_{n \in \omega} C_{n}
$$


where

$$
C_{n}= \begin{cases}A_{n} & \text { if } n \in S \\ I & \text { otherwise }\end{cases}
$$

For $S, T \subseteq \omega$ and any function $\xi: S \rightarrow T$, define $\xi_{*}: \mathscr{A}^{S} \rightarrow \mathscr{A}^{T}$ by

$$
\xi_{*}(A)_{n}=\otimes_{\xi(m)=n} A_{m}
$$

for all $A \in \mathscr{A}^{S}$ and $n \in T$.

Definition 10.1. An $\omega$-monoidal category is a category $\mathscr{A}$ equipped with an object $I \in \mathscr{A}$, a functor

$$
\otimes: \mathscr{A}^{\omega} \longrightarrow \mathscr{A},\left(A_{i}\right)_{i \in \omega} \longmapsto \otimes_{i \in \omega} A_{i}
$$

and, for all order-preserving functions $\xi: \omega \rightarrow \omega$, natural isomorphisms
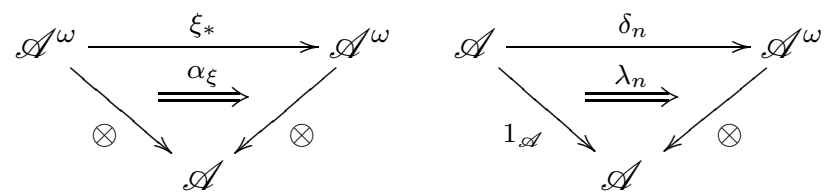

subject to the conditions that the components of the $\lambda_{n}$ at $I$ are all equal and there are the equations (10.3) and (10.4) of pasting diagrams.
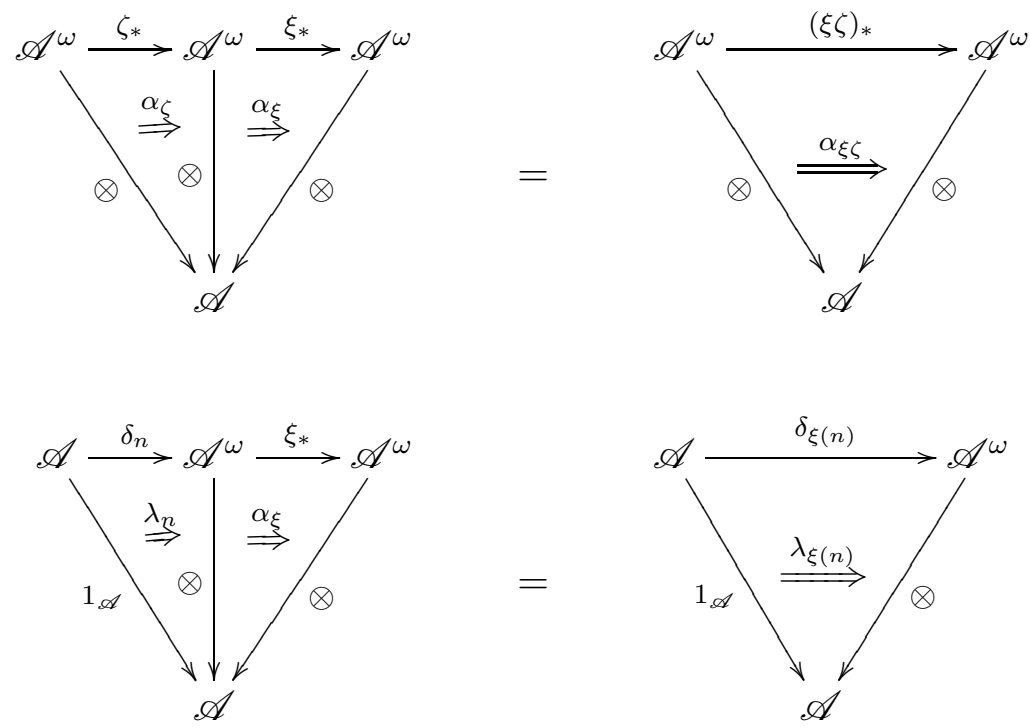

Example 10.2. Every series monoidal category is $\omega$-monoidal with $\otimes=\Sigma$ and $I=0$. 
Example 10.3. As a categorical version of Example 9.5, if $\mathscr{A}$ is a "series rig category", possibly without unit, then $\mathscr{A}$ becomes $\omega$-monoidal with $\otimes$ taken to be a categorical version of the $\mathrm{P}$ of $(3.1)$ and $I=0$.

\section{References}

[1] Aristotle, Physics 6(9) (350 B.C.E) $239 \mathrm{~b} 10$.

[2] Jean Bénabou, Introduction to bicategories, Lecture Notes in Mathematics 47 (Springer-Verlag, 1967) 1-77.

[3] Robert Blackwell, G. Max Kelly and A. John Power, Two-dimensional monad theory, J. Pure Appl. Algebra 59 (1989) 1-41.

[4] Brian Day, *-Autonomous categories in quantum theory, www. arxiv.org/abs/math/0605037 5 pp.

[5] Brian Day and Ross Street, Monoidal bicategories and Hopf algebroids, Advances in Math. 129 (1997) 99-157.

[6] Samuel Eilenberg and G.M. Kelly, Closed categories, Proceedings of the Conference on Categorical Algebra (La Jolla, 1965), (SpringerVerlag,1966) 421-562.

[7] J. Fillmore, D. Pumplün and H. Röhrl, On N-summations, I, Applied Categorical Structures 10 (2002) 291-315.

[8] Peter Freyd, Algebraic real analysis, Theory and Applications of Categories, 20(10) (2008) 215-306.[8]

[9] Denis Higgs, A universal characterization of $[0, \infty]$, Nederl. Akad. Wetensch. Indag. Math. 40(4) (1978) 448-455.

[10] Denis Higgs, Axiomatic infinite sums - an algebraic approach to integration theory, Contemp. Math. 2 (Amer. Math. Soc., 1980) 205-212.

[11] E.V. Huntingdon, A complete set of postulates for the theory of absolute continuous magnitude, Transactions Amer. Math. Soc. 3 (1902) 264279 .

[12] André Joyal and Ross Street, The geometry of tensor calculus, I, Advances in Mathematics 88 (1991) pp. 55-112. 
[13] André Joyal, Ross Street and Dominic Verity Traced monoidal categories, Mathematical Proceedings of the Cambridge Philosophical Society 119(3) (1996) 425-446.

[14] G.M. Kelly, Many-variable functorial calculus I, Lecture Notes in Math. 281 (Springer-Verlag, 1972) 66-105.

[15] G.M. Kelly, Doctrinal adjunction, Lecture Notes in Mathematics 420 (Springer-Verlag, 1974) 257-280.

[16] G.M. Kelly, Basic concepts of enriched category theory, London Mathematical Society Lecture Note Series 64 (Cambridge University Press, Cambridge, 1982).

[17] G.M. Kelly and Ross Street, Review of the elements of 2-categories, Lecture Notes in Mathematics 420 (Springer-Verlag, 1974) 75-103.

[18] Anders Kock, Closed categories generated by commutative monads, J. Australian Math. Soc. 12 (1971) 405-424.

[19] F. W. Lawvere, Metric spaces, generalized logic and closed categories, Reprints in Theory and Applications of Categories 1 (2002) pp.1-37; originally published as: Rendiconti del Seminario Matematico e Fisico di Milano 53 (1973) 135-166.

[20] Saunders Mac Lane, Categories for the Working Mathematician, Graduate Texts in Mathematics 5 (Springer-Verlag, 1971).

[21] Chi-Keung Ng, On genuine infinite algebraic tensor products, Revista Matemática Iberoamericana, 29(1) (2013) 329-356.

[22] Stephen H. Schanuel, Negative sets have Euler characteristic and dimension, Lecture Notes in Mathematics 1488 (Springer, 1991) 379385 .

[23] Zbigniew Semadeni, Monads and their Eilenberg-Moore algebras in functional analysis, Queen's Papers in Pure and Applied Mathematics 33 (Queen's University, Kingston, Ont., 1973) iii+98 pp.

[24] Ross Street, Fibrations in bicategories, Cahiers de topologie et géométrie différentielle 21 (1980) 111-160.

[25] Ross Street, An efficient construction of the real numbers, Gazette Australian Math. Soc. 12 (1985) 57-58; also see http://maths.mq.edu.au/ street/EffR.pdf . 
[26] Ross Street, Skew-closed categories, Journal of Pure and Applied Algebra 217(6) (2013) 973-988.

[27] Ross Street, Weighted tensor products of Joyal species, graphs, and charades, SIGMA 12(005) (2016) 20pp.

[28] Alfred Tarski, Cardinal algebras (New York, Oxford University Press, 1949).

[29] Alfred Tarski, Ordinal algebras (North-Holland, Amsterdam, 1956).

[30] Stephen T. Welstead, Infinite products in a Banach algebra, Journal of Math. Analysis and Applications 105 (1985) 523-532. 